\section{Médecin et armée}

L'armée manque de médecins militaires. Ce point critique - et chronique - est connu de tous ceux qui ont organisé des cours ou des exercices militaires ces vingt dernières années. Les raisons en sont multiples. La mutation des valeurs sociétales a généré des problèmes dans l'armée tout entière. L'aura conférée par la fonction militaire a pâli. A cela s'ajoute que dans le service sanitaire, l'intention d'harmoniser, dans un laps de temps raisonnable, les études de médecine et la formation postgraduée avec la carrière de médecin militaire se révèle une entreprise des plus ardues, lourde de sacrifices. Les compensations encourageant ce type de carrière ont été jusqu'ici bien timides, voire inexistantes.

Pour l'étudiant en médecine, les fins de semestres et les examens contrecarrent les écoles et les cours militaires. Le médecin assistant aux ambitions militaires a plus de peine que d'autres de trouver des places de formation appropriées. La charge pour les hôpitaux que représente, sur le plan économique et d'organisation, la recherche de places de formation pour les personnes soumises aux obligations militaires, est devenue un critère de sélection prioritaire.

On relève également un manque de motivation sur le plan professionnel, les qualifications acquises au service militaire ne recueillant ni reconnaissance, $n i$ prise en compte.

L'intervention de plus en plus marquée de l'Etat dans l'exercice de la profession médicale, avec sa politique tarifaire qui depuis longtemps ne correspond plus à l'évolution des coûts de la petite entreprise qu'est un cabinet médical, a pour conséquence de détruire toute velléité d'engagement dans l'armée au delà du strict nécessaire. Crise de confiance et perte de motivation sont désormais le lot de confrères qui prônaient, précisément, l'engagement à l'égard de la collectivité. L'armée y a également contribué. En effet, pour pallier le manque de médecins militaires, elle s'est fondée sur des dispositions légales, certes, mais "manu militari", pour obliger d'anciens officiers sanitaires à accomplir des périodes supplémentaires de service. Ce ne sont pas là précisément des méthodes aptes à encourager la relève.

Le résultat de cette évolution est là: seuls $10 \%$ des étudiants en terminale sont prêts à s'engager dans une formation de médecin militaire et la volonté de servir dans des fonctions de cadre supérieur est en voie de disparition.

Le plan directeur de l'armée XXI et la révision de la loi militaire, qui ont été mis en consultation jusqu'au 31 juillet 2001, font craindre que la situation ne se perpétue. La diminution de la durée du service militaire obligatoire en général ne fait qu'aggraver les problèmes. Le projet visant à supprimer les troupes sanitaires en tant que telles et à les intégrer dans les troupes logistiques a suscité de vives réactions au sein du corps médical. Cette solution est diamétralement opposée aux besoins des patients, aux tâches qui relèvent $d u$ service sanitaire coordonné et aux exigences d'une formation de haut niveau du personnel sanitaire. Elle est dès lors inacceptable.

A l'émergence d'Armée XXI, nous avons mis au point des revendications et des exigences, mais aussi des propositions, élaborées avec la Commission interfacultés médicale suisse (CIMS). Nous attendons du projet Armée XXI qu'il ait des égards particuliers non seulement pour les chevaux du train, mais aussi pour les médecins militaires et le service sanitaire!

Dr Reto Laetsch Membre du Comité central de la FMH

\title{
Communication importante
}

Selon une decision du Comité central de la FMH prise le 15 septembre 2001, le 25 octobre 2001 aura lieu une Conférence des Présidents, et non pas, comme cela avait été annoncé dans le BMS No 37 (p. 1953), une Chambre médicale extraordinaire; celle-ci est reportée à décembre. La Conférence des Présidents du 26 septembre 2001 n'est pas touchée par ce changement. Des informations supplémentaires suivront, voir aussi www.fmh.ch. 